\title{
Assuring quality in Canada's microbiology laboratories
}

$\mathrm{T}^{1}$ is issue of the Journal presents three articles from two Canadian laboratory proficiency testing programs, the Ontario Laboratory Proficiency Testing Program and the University of British Columbia's Clinical Microbiology Proficiency Testing Program. Two papers examine the various facets of performing urine cultures and reporting results (Richardson et al, pages 243-246 and Noble et al, pages 247-249). Both found that Canadian laboratories use inconsistent units when quantifying organisms in urine. The third paper demonstrates that many laboratories examining stool for ova and parasites do very low volumes of testing and many have unacceptably long turnaround times (Richardson et al, pages 237-242). Both common sense and previously published papers have suggested that parasitology is best done by experienced technologists (1). Such experience can only be gained in a laboratory processing an adequate volume of positive specimens.

Clinicians reading these articles will ask, "To what extent can these observations be extrapolated to other areas of diagnostic testing?" Can clinicians be assured that both simple and highly complex laboratory testing are clinically relevant, and that results are accurate, reported in a timely fashion and generated in a manner consistent among laboratories? Such questions may be increasingly relevant in an era of cost containment and restructuring in our health care system. Are the dramatic changes in many of our nation's laboratories likely to be reflected in deteriorating quality of diagnostic testing?

While the submission of specimens for proficiency testing may be useful, at best, the results represent an insensitive surrogate marker of overall laboratory performance. Proficiency testing samples differ from routinely submitted clinical specimens; therefore, both pre-analytic (collection, transport, assessment for quality and suitability) and postanalytic (timeliness and formatting of reports) aspects of testing may not be assessed. Many laboratories choose not to blind staff to the nature of the specimen; as a result, it is often subject to special treatment. The papers contained in this issue reflect the desire to broaden the scope of programs beyond that of conventional proficiency testing in order to examine more complex processes.

There is a compelling need to examine how quality in laboratories can be improved. Once the technical development of a new method has been accomplished, it is just as important to understand how testing of clinical specimens should be performed for the appropriate patients, and the limitations of the results of that testing. Many questions will need to be answered if we are to understand better the true determinants of quality. How do the qualifications of professional and technical staff relate to the quality of testing? What is the appropriate extent of testing as it relates to different specimen types? How can proficiency testing programs be improved in order to measure all stages of specimen handling, testing and reporting? How can proficiency testing be improved to identify problems most likely directly to affect patient care? How can new programs be designed to monitor emerging technologies better, especially in the field of molecular diagnostics?

The accumulated knowledge may, in the long term, allow us to develop new or improved guidelines for the conduct of laboratory services. It is hoped that our professional societies will accept the challenge and, by working together, produce guidelines that will ultimately improve patient care. While some may argue that these guidelines will eventually become regulations in our increasingly regulated environment, I would argue that this process is unlikely to increase the pace of regulation. It may be better to have the profession's own guidelines adopted by regulators rather than the reverse. Canada's proficiency programs should begin to examine some of the broader issues, and I challenge our professional societies to set aside differences and to focus their energy and expertise on developing a national program to assist laboratories to meet our high expectations.

\section{REFERENCE}

1. Mohr E, Mohr I. Statistical analysis of the incidence of positives in the examination of parasitological specimens. J Clin Microbiol $1992 ; 30: 1572-4$

Kevin R Forward MD FRCPC Service Chief, Division of Microbiology Department of Pathology and Laboratory Medicine Queen Elizabeth II Health Sciences Centre Halifax, Nova Scotia 


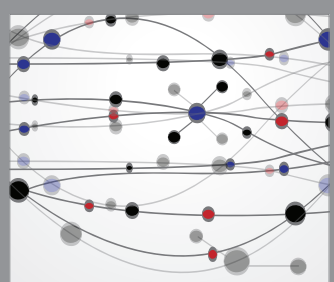

The Scientific World Journal
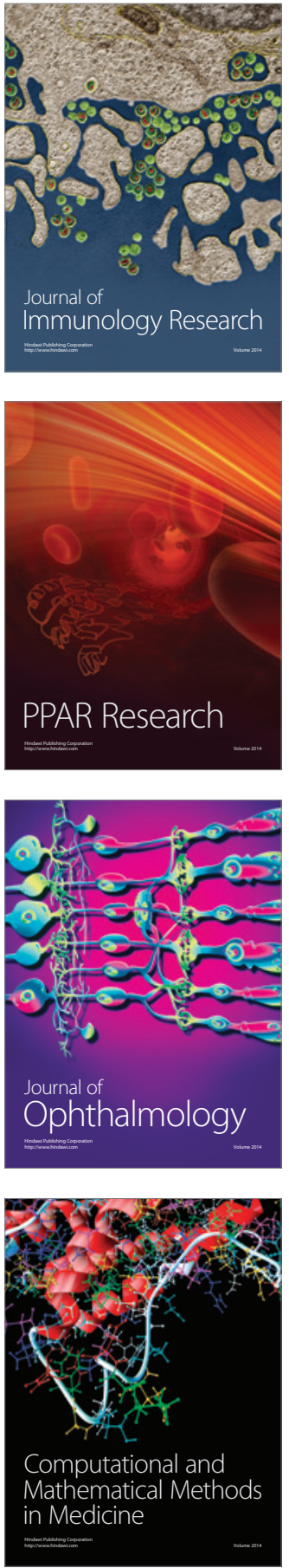

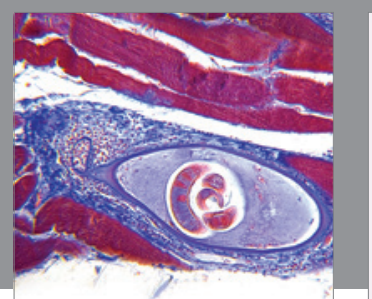

Gastroenterology Research and Practice

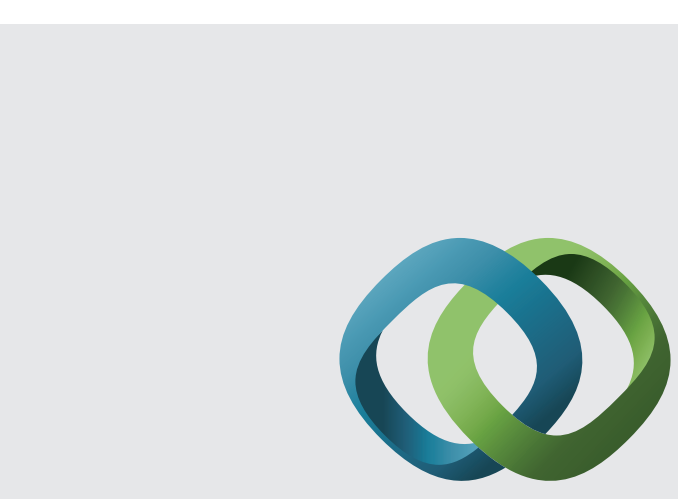

\section{Hindawi}

Submit your manuscripts at

http://www.hindawi.com
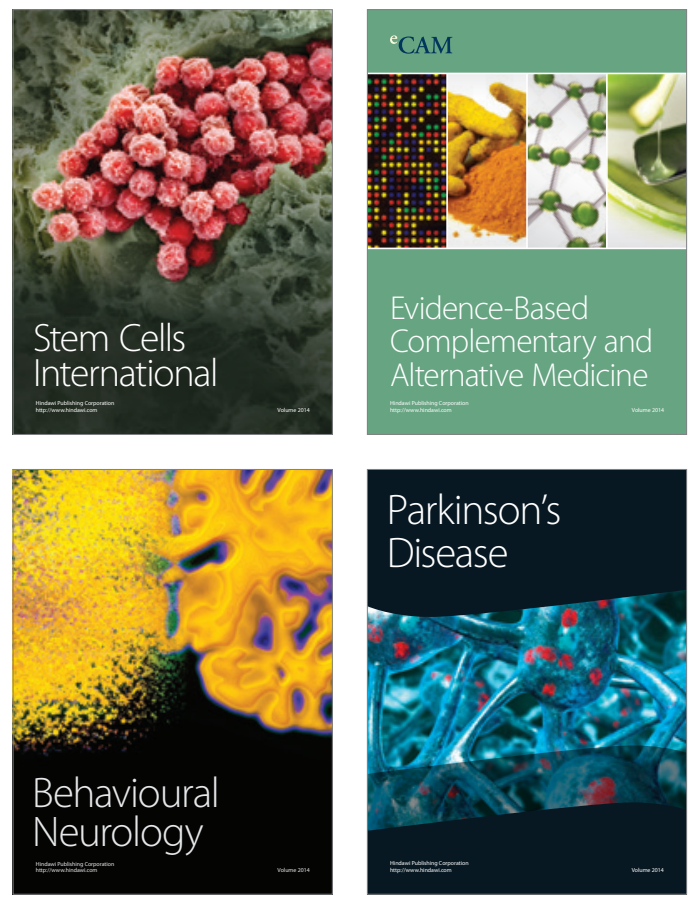
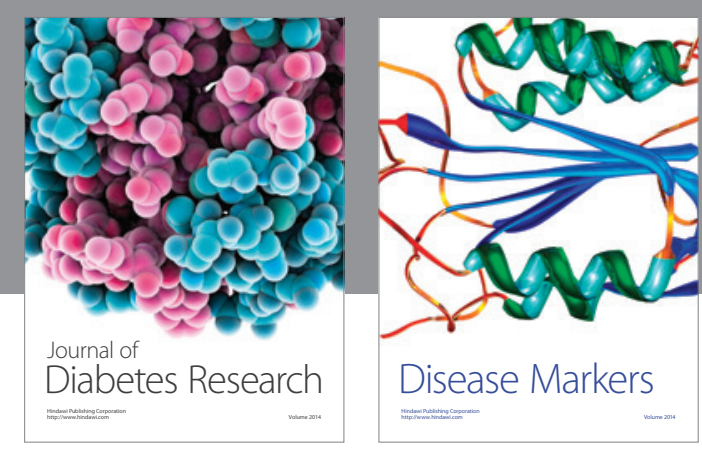

Disease Markers
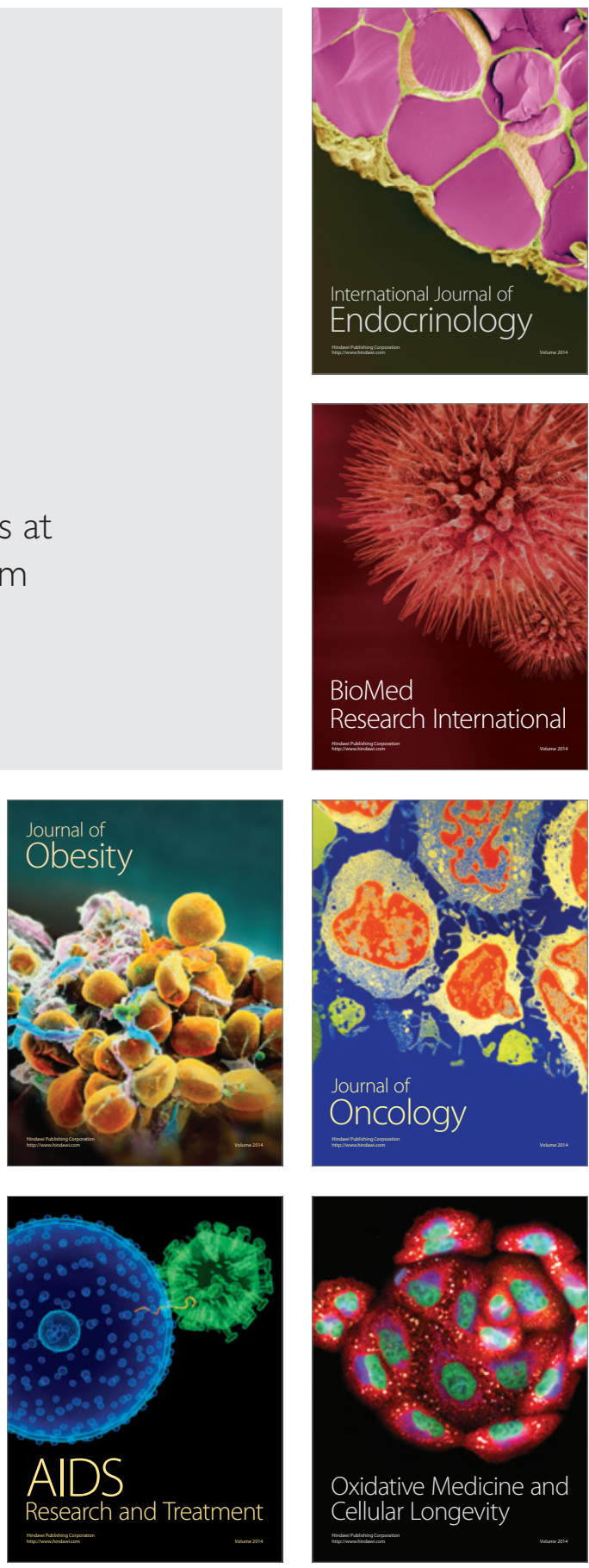